\title{
ORTHOGONAL DISCRETE FREQUENCY CODING SPACE TIME WAVEFORM FOR MIMO RADAR DETECTION IN COMPOUND GAUSSIAN CLUTTER
}

\author{
B. Roja Reddy ${ }^{1}$ and M. Uttarakumari ${ }^{2}$ \\ ${ }^{1}$ Department of Telecommunication Engineering, \\ R.V. College of Engineering, Bangalore, India. \\ rojareddybervce.edu. in \\ ${ }^{2}$ Department of Electronics and Communication Engineering, \\ R.V. College of Engineering, Bangalore, India \\ uttarakumariervce.edu. in
}

\begin{abstract}
This paper proposes orthogonal Discrete Frequency Coding Space Time Waveforms (DFCSTW) for Multiple Input and Multiple Output (MIMO) radar detection in compound Gaussian clutter. The proposed orthogonal waveforms are designed considering the position and angle of the transmitting antenna when viewed from origin. These orthogonally optimized show good resolution in spikier clutter with Generalized Likelihood Ratio Test (GLRT) detector. The simulation results show that this waveform provides better detection performance in spikier Clutter.
\end{abstract}

\section{KEYWORDS}

Multiple Input and Multiple Output (MIMO), orthogonal Discrete Frequency Coded Space Time Waveforms (DFCSTW), Generalized Likelihood Ratio Test (GLRT), Compound Gaussian Clutter

\section{INTRODUCTION}

Multiple Input and Multiple Output system (MIMO) transmits multiple linearly independent probing signals via its transmit antennas and receives multiple coded waveforms from multiple locations. MIMO radar systems have many advantages including increased angle resolution [1-6], increased Doppler resolution [1,7], reduced ground-based radar clutter levels [1], sharper airborne radar clutter notches [2,7], Lower Probability of Intercept (LPI) [1,8], and relaxed hardware requirements [1].

The performance of the transmitted waveforms is judged by their correlation properties [9-12]. The waveforms should have good autocorrelation properties for high range resolution and good cross-correlation for multiple target return separability. So, there is a need to design MIMO radar waveforms as orthogonal pulses with low correlation properties. In literature various algorithms have been proposed to design the orthogonal sequences with low autocorrelation and crosscorrelation peak sidelobe levels. In [9], the focus is to design orthogonal Discrete Frequency Coding Waveforms_Frequency Hopping (DFCW_FF) for netted radar systems using simulated

Natarajan Meghanathan et al. (Eds) : ICCSEA, SPPR, VLSI, WiMoA, SCAI, CNSA, WeST - 2014 pp. 59-66, 2014. (C) CS \& IT-CSCP 2014

DOI : $10.5121 /$ csit.2014.4714 
annealing (SA) algorithm to optimize the frequency sequences, [10] and [11] focus on orthogonal DFCW_FF and orthogonal Discrete Frequency Coding Waveforms_Linear Frequency Modulation (DFCW_LFM) to design multiple orthogonal sequences with good correlation using a Modified Genetic Algorithm (MGA) technique. In [12] various Cyclic Algorithms (CA) for unimodular MIMO radar waveforms are designed for good correlation properties. Target Radar Cross Section (RCS) fades the received signal from the target. One way to maximize the system's processing gain is by antenna spacing [13-15]. In [16], adaptive space time waveform was proposed to improve detection performance. To improve the system performance, antenna spacing is one of the important factors.

Statistical characterization of the clutter is necessary for designing the detector. The clutter echoes result from very large number of elementary scatterers due to which the radar system has relatively low resolution capability. As the radar resolution increases, the statistics of the clutter has no longer been observed to be Gaussian. There is experimental evidence that high resolution radar systems are now plagued by target-like "spikes" that gives rise to non-Gaussian heavy tailed observations [17-18]. The high resolution sea clutter is modelled by compound Gaussian clutter which is a sample of compound $\mathrm{K}$-distribution clutter. In [19-23] the focus is on the Generalized Likelihood Ratio Test (GLRT) detector to yield excellent performance and it is very much attractive for radar detection in the presence of correlated non-Gaussian clutter model.

In this paper, the proposed Orthogonal Discrete Frequency Coded Space Time Waveforms (DFCSTW) can improve the target detection in compound Gaussian (spikier) clutter. Section II illustrates the signal model and GLRT detector, section III shows the numerical simulations for the model developed in section II. Section IV concludes the paper.

\section{WAVEFORM MODEL}

Consider a MIMO radar system with a $T_{x}$ transmitting antennas. Let $x_{i}$ where $i=\left\{1,2, \ldots T_{x}\right.$ ) denote the position of $\mathrm{T}_{\mathrm{x}}$ transmitting antennas located at an angle $\theta_{\mathrm{i}}$ when viewed from an origin. Each element may transmit $\mathrm{N}$ coding frequencies on each subpulse of a waveform. Each $\mathrm{R}_{\mathrm{x}}$ receives and processes the signal from all the $T_{x}$ transmitters. The received signals are the reflected signals from a target and clutter. Each element transmits $\mathrm{N}$ pulses with a Pulse Repetition frequency (PRF) of $\mathrm{f}_{\mathrm{r}}$.

\subsection{Discrete Frequency Coding Space Time Waveform (DFCSTW)}

Linear Frequency Modulation (LFM) is the first and probably the most popular pulse compression method. Discrete Frequency-coding Waveform (DFCW) has the large compression ratio. DFCW can lower correlation properties if sequences are coded properly. The basic idea is to sweep the frequency band (B) linearly during the pulse duration (T) and the time bandwidth product of the signal is BT. The spectral efficiency of the DFCW improves as the time-bandwidth product increases, because the spectral density approaches a rectangular shape. Here we consider the sequence length of each waveform $(\mathrm{N})$ and Number of antennas $\left(\mathrm{T}_{\mathrm{x}}\right)$.

The Discrete Frequency Coding Space Time (DFCSTW) Waveform is defined as

$$
\mathrm{S}_{\mathrm{p}}(\mathrm{t}, \Phi)=\left\{\begin{array}{ll}
\sum_{\mathrm{n}=0}^{\mathrm{N}-1} \mathrm{e}^{\mathrm{j} 2 \pi \mathrm{f}_{\mathrm{n}}^{\mathrm{p}}\left(\mathrm{t}-\mathrm{nT}_{\mathrm{p}}\right)} \mathrm{e}^{\mathrm{j} \pi \mathrm{kt}^{3}} \mathrm{e}^{\mathrm{j} 2 \pi \mathrm{x}_{\mathrm{p}} \sin \Phi_{\mathrm{p}} \mathrm{f}_{\mathrm{k}} / \mathrm{cf}}, 0 \leq \mathrm{t} \geq \mathrm{T}_{\mathrm{p}} \\
0 & , \text { elsewhere }
\end{array}\right\}^{\mathrm{T}}, \mathrm{p}=1,2 \ldots \mathrm{T}_{\mathrm{x}}
$$


where $\mathrm{s}$ is the frequency slope, $\mathrm{s}=\mathrm{B} / \mathrm{T}$ and $\mathrm{p}=1,2, \ldots, \mathrm{T}_{\mathrm{x}} . \mathrm{T}$ is the subpulse time duration. $\mathrm{N}$ is the number of subpulse that is continuous with the coefficient sequence $\left\{\mathrm{n}_{1}, \mathrm{n}_{2}, \ldots \ldots . \mathrm{n}_{\mathrm{Tx}}\right\}$ with unique permutation of sequence $\{0,1,2, \ldots \ldots N\} . f_{n}{ }^{p}=n \Delta f$ is the coding frequency of subpulse $n$ of waveform $\mathrm{p}$ in the waveform. $\Delta \mathrm{f}$ is the frequency step. Where $\mathrm{x}_{\mathrm{p}}, \mathrm{i}=\left\{1,2, \ldots \mathrm{T}_{\mathrm{x}}\right)$ denote the position of $T_{x}$ transmitting antennas located at an angle $\theta_{i}$ when viewed from an origin. The choice of BT, $\mathrm{T} . \Delta \mathrm{f}$ and $\mathrm{B} / \Delta \mathrm{f}$ values are crucial for the waveform design. Different lengths of firing sequence $(\mathrm{N})$ have different values for each of the above mentioned parameters [11].

\section{SIGNAL MODEL}

The received signals for MIMO radar can be formulated as

$$
\mathrm{r}_{\mathrm{i}}=\mathrm{S}_{\mathrm{i}} * \mathrm{~T}_{\mathrm{i}}+\mathrm{S}_{\mathrm{i}} * \mathrm{Cl}_{\mathrm{i}}+\mathrm{V}_{\mathrm{i}}, \mathrm{i}=1,2, \ldots \mathrm{R}_{\mathrm{x}}
$$

Where $\mathrm{S}$ is the transmitted code matrix. $\mathrm{T}_{\mathrm{i}}=\left[\mathrm{T}_{\mathrm{i}}, \ldots \ldots \ldots, \mathrm{T}_{\mathrm{iTx}}\right]^{\mathrm{T}}, \mathrm{i}=1,2, \ldots \ldots \mathrm{R}_{\mathrm{x}}$ are the complex values accounting for both the target backscattering. $\mathrm{V}=\left[\mathrm{V}_{\mathrm{i} 1}, \ldots \ldots \ldots, \mathrm{V}_{\mathrm{iTx}}\right]^{\mathrm{T}}, \mathrm{i}=1,2, \ldots \ldots \mathrm{R}_{\mathrm{x}}$ are noise component. $r_{i}=\left[r_{i 1}, \ldots \ldots \ldots, r_{i N}\right]^{T}, i=1,2 \ldots \ldots . . R x$ are the echo signals of the $i^{\text {th }}$ receiver antenna contaminated by the clutter. The clutter vectors $n_{i}$ are assumed as compound Gaussian random vector i.e., [22]

$$
\mathrm{Cl}_{\mathrm{i}}=\sqrt{\alpha_{\mathrm{i}} \beta_{\mathrm{i}}}, \mathrm{i}=1, \ldots \ldots \ldots \ldots \mathrm{R}_{\mathrm{x}}
$$

The texture $\alpha_{\mathrm{i}}$ is non-negative random variable which models the variation in power that arises from the spatial variation in the backscattering of the clutter and the speckle components $\beta_{\mathrm{i}}$ are correlated complex circular Gaussian vectors and independent to one other. This $\alpha_{i}$ is independent Zero-mean complex circular Gaussian vector with covariance matrix.

$$
\mathrm{R}_{\mathrm{i}}=\mathrm{E}\left[\mathrm{n}_{\mathrm{i}} \mathrm{n}_{\mathrm{i}}^{\mathrm{H}}\right]=\alpha_{\mathrm{i}} \mathrm{r}_{\mathrm{o}}
$$

Whereas $r_{0}=\left[\mathrm{Cl}_{i} \mathrm{Cl}_{\mathrm{i}}^{\mathrm{H}}\right]$ is the covariance structure and $\mathrm{H}$ is complex conjugate. The Compound Gaussian clutter is samples from K-distribution with pdf.

$$
f(z)=\frac{\sqrt{2 v / \mu}}{\tau(v)}\left(\sqrt{\frac{2 v}{\mu}} z\right)^{v} k_{v-1}\left(\sqrt{\frac{2 v}{\mu}} z\right)
$$

The texture component $\sqrt{\tau_{i}}$ is gamma distribution with pdf

$$
f\left(\tau_{i}\right)=\frac{1}{\sqrt{(v)}}\left(\frac{v}{\mu}\right)^{v}{\sqrt{\tau_{i}}}^{v-1} e^{-v / \mu \sqrt{\tau_{\mathrm{i}}}} u\left(\sigma_{i}\right)
$$

where $\Gamma$ (.) is the Eulerian Gamma function, $v>0$ is the parameter ruling the shape of the distribution, $\mathrm{u}($.$) denotes the unit-step function, and \mathrm{K}_{\mathrm{v}}($.$) is the modified second kind Bessel$ function with order $v$, which rules the clutter spikiness. The smaller the value of $v$, the higher is the tails of the distribution. The distribution will become Gaussian for $v \rightarrow \infty$.

The clutter has exponential correction structure of covariance matrix $r_{0}$, the $(i, j)$ element of which is $\rho^{\mathrm{li}-\mathrm{jl},}$ where $\rho$ is one-lag correlation coefficient. The Power Spectral Density of clutter is 
generally located in low frequency region \& Clutter spread is controlled by v. The small the values of $\mathrm{v}$ the spikier is the clutter.

\subsection{GLRT Detector}

Suppose $k(k \geq N)$ secondary data vector, sharing the same covariance structure of the primary data is available, $r_{i}$ and $r_{i k}, i=1,2 \ldots . R_{x}, k=1,2 \ldots k$ are the received signal from the primary and secondary data. Then, the detecting of a target with MIMO radar can be formulated in terms of the following binary hypotheses test.

$$
\begin{aligned}
& \mathrm{H}_{0}:\left\{\begin{array}{l}
\mathrm{r}_{\mathrm{i}}=\mathrm{S}_{\mathrm{i}} * \mathrm{Cl}_{\mathrm{i}}+\mathrm{V}_{\mathrm{i}}, \mathrm{i}=1,2, \ldots \ldots \mathrm{R}_{\mathrm{x}} \\
\mathrm{r}_{\mathrm{ik}}=\mathrm{S}_{\mathrm{ik}} * \mathrm{Cl}_{\mathrm{ik}}+\mathrm{V}_{\mathrm{ik}}, \mathrm{i}=1,2, \ldots \ldots . \mathrm{R}_{\mathrm{x}}, \mathrm{k}=1,2, \ldots \ldots \mathrm{k}
\end{array}\right. \\
& \mathrm{H}_{1}:\left\{\begin{array}{l}
\mathrm{r}_{\mathrm{i}}=\mathrm{S}_{\mathrm{i}} * \mathrm{~T}_{\mathrm{i}}+\mathrm{S}_{\mathrm{i}} * \mathrm{Cl}_{\mathrm{i}}+\mathrm{V}_{\mathrm{i}}, \mathrm{i}=1,2, \ldots \ldots \mathrm{R}_{\mathrm{x}} \\
\mathrm{r}_{\mathrm{ik}}=\mathrm{S}_{\mathrm{ik}} * \mathrm{~T}_{\mathrm{ik}}+\mathrm{S}_{\mathrm{ik}} * \mathrm{Cl}_{\mathrm{ik}}+\mathrm{V}_{\mathrm{ik}}, \mathrm{i}=1,2, \ldots \ldots \mathrm{R}_{\mathrm{x}}, \mathrm{k}=1,2, \ldots \ldots \mathrm{k}
\end{array}\right.
\end{aligned}
$$

The GLRT detector [22] based on the primary data can be obtained by replacing the unknown parameters with their maximum likelihood estimates in the likelihood ratio. The GLRT detector [22] of the complex amplitude TH

$$
\begin{aligned}
& \mathrm{H}_{1} \\
& \prod_{i=1}^{\mathrm{r}} \frac{\mathrm{r}_{\mathrm{i}}^{\mathrm{H}} \mathrm{r}_{\mathrm{o}}^{-1} \mathrm{r}_{\mathrm{i}}}{\mathrm{r}_{\mathrm{i}}^{\mathrm{H}}\left(\mathrm{r}_{\mathrm{o}}^{-1}-\mathrm{r}_{\mathrm{o}}^{-1} \mathrm{~S}\left(\mathrm{~S}^{\mathrm{H}} \mathrm{r}_{\mathrm{o}}^{-1} \mathrm{~S}^{-1} \mathrm{~S}^{\mathrm{H}} \mathrm{r}_{\mathrm{o}}^{-1}\right) \mathrm{r}_{\mathrm{o}}\right.}>\mathrm{TH} \\
& \mathrm{H} \text { 。 }
\end{aligned}
$$

where the TH is variable detection threshold. In a practical adaptive radar system, the covariance matrix of the clutter is estimated from a set of secondary data, which must be representative of the samples in the Cell Under Test (CUT). To make the detectors ensure the CFAR property w.r.t texture statistics, a normalized sample covariance matrix is adopted, based on the secondary data collected by the receiver antennas.

$$
\hat{\mathrm{R}}_{\mathrm{oi}}=\frac{\mathrm{N}}{\mathrm{K}} \sum_{\mathrm{k}=1}^{\mathrm{k}} \frac{\mathrm{n}_{\mathrm{i}, \mathrm{k}} \mathrm{n}_{\mathrm{i}, \mathrm{k}}^{\mathrm{H}}}{\mathrm{n}_{\mathrm{i}, \mathrm{k}}^{\mathrm{H}} \mathrm{n}_{\mathrm{i}, \mathrm{k}}}
$$

Substituting eq. (9) in eq. (8), we get the adaptive detector.

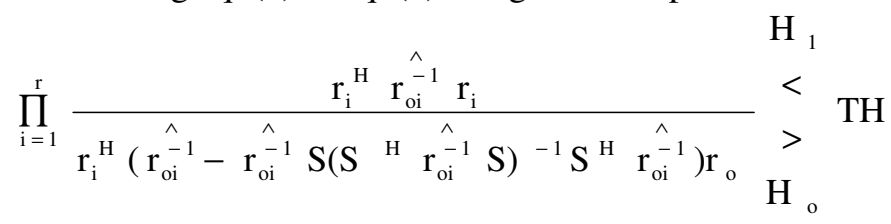

For a given value of $\mathrm{N}$, as $\mathrm{k}$ varies the proposed adaptive detectors end up coincident with real scenario. However, for finite values of $\mathrm{K}$, the performance of the estimate and eventually of the adaptive detector itself depends upon the actual values of $\mathrm{N}$. Thus it is necessary to quantify the loss of the proposed decision strategy with respect to its non adaptive counterpart under situations of exact covariance matrix.

In order to compare the performance of the GLRT detector with Gaussian clutter GLRT detector (GC-GLRT),

$$
\sum_{\mathrm{i}=1}^{\mathrm{r}} \mathrm{r}_{\mathrm{i}} \hat{\mathrm{r}}_{\mathrm{oi}}^{-1} \mathrm{~S}\left(\mathrm{~S}^{\mathrm{H}} \mathrm{r}_{\mathrm{oi}}^{-1} \mathrm{~S}\right)^{-1} \mathrm{~S}^{\mathrm{H}} \stackrel{\mathrm{r}_{\mathrm{oi}}^{-1} \mathrm{r}_{\mathrm{i}}}{>} \mathrm{TH}
$$


In order to limit the computational burden, we assume $\mathrm{P}_{\mathrm{fa}}$ as $10^{-4}$ and also to save the simulation time. The transmit code matrix S is the orthogonal DFCSTW and the Signal-to-Clutter Ratio (SCR) is defined as

$$
\mathrm{SCR}=\frac{\alpha^{2}}{\mathrm{NT}_{\mathrm{x}}} \operatorname{tr}\left[\mathrm{S}^{\mathrm{H}} \mathrm{r}_{\mathrm{oi}}^{-1} \mathrm{~S}\right]
$$

\section{DESIGN RESULTS}

Consider an orthogonal DFCSTW code set for $4 * 4$ MIMO radar with code length of $\mathrm{N}=8$. The simulation is carried out in MATLAB. The frequency code sets are optimized using ACC_PSO [24]. These sequences are considered in eq. (1) to generate the orthogonal DFCSTW set with good correlation properties. The above generated Code sequence matrix is used in the signal model mentioned in the section II to generate the DFCSTW where $\theta_{\mathrm{i}}$ is the angle of the $\mathrm{T}_{\mathrm{x}}$ transmitting antennas are generated randomly and placed linearly. Thus generated waveform is implemented in the signal model mentioned in the section III.

The $\mathrm{P}_{\mathrm{ds}}$ of GLRT and of GC-GLRT are plotted versus SCR with $\mathrm{P}_{\mathrm{fa}}=10^{-4}, \mathrm{~N}=8, \mathrm{~N}_{\mathrm{T}}=4, \mathrm{~N}_{\mathrm{R}}=4$, $\rho=0.9, \mathrm{~K}=64, \mathrm{v}=0.5$ in Fig.1. The performance of GLRT is better than GC-GLRT. Fig 2 shows the pds versus SCR for orthogonal DFCSTW code set and Space Time Code (STC) waveforms. It can be observed that the performance of orthogonal DFCSTW code is better than STC. The value of Pds is 0.6 at $-25 \mathrm{~dB}$ in [21] and the simulated result is 0.68 at the same $\mathrm{dB}$ value for DFCSTW code. This shows that the orthogonal DFCSTW code sets perform better in the spikier clutter than STC.

Fig 3 shows the pds versus SCR for orthogonal DFCSTW code set for different values of v. As the values of $\mathrm{v}$ decreases the clutter is spikier and the detector works better of the value of $\mathrm{v}=0.3$ than $\mathrm{v}=0.8$. Thus the DFCSTW code set has better detection performance in spikier environment. In Table 1 , lists the values of Pds versus SCR for $-10 \mathrm{~dB}$. It can be observed that the performance of this paper is much better than the existing statistics [20-22]. The curves show that the performance of GLRT with orthogonal DFCSTW code set is better in spikier clutter.

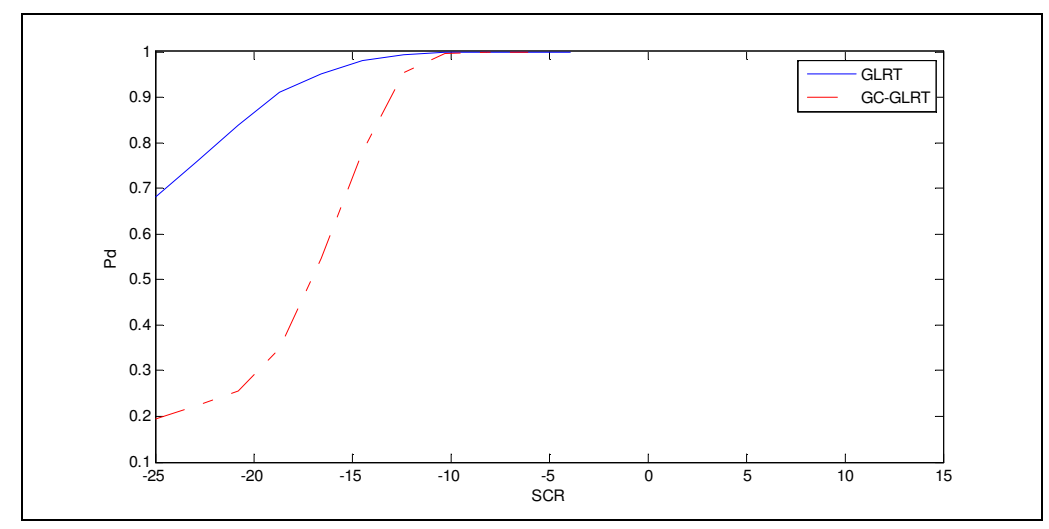

Figure 1. Pd versus SCR plots of GLRT (solid curves) and GCGLRT (dashed curves) receivers with Orthogonal DFCSTW Code set for $\mathrm{Pfa}=10^{-4}, \mathrm{~N}=8, \mathrm{Tx}=4, \mathrm{Rx}=4, \rho=0.9, \mathrm{~K}=64$, v=0.5 parameter. 
TABLE 1. Values of Pd Vs SCR

\begin{tabular}{|l|l|l|}
\hline Literature & SCR (in dB) & \multicolumn{1}{|c|}{ Pd } \\
\hline$[20]$ & -10 & 0.02 \\
\hline$[21]$ & -10 & 0.95 \\
\hline$[22]$ & -10 & 0.8 \\
\hline $\begin{array}{l}\text { For the } \\
\text { simulated } \\
\text { result }\end{array}$ & -10 & 0.998 \\
\hline
\end{tabular}

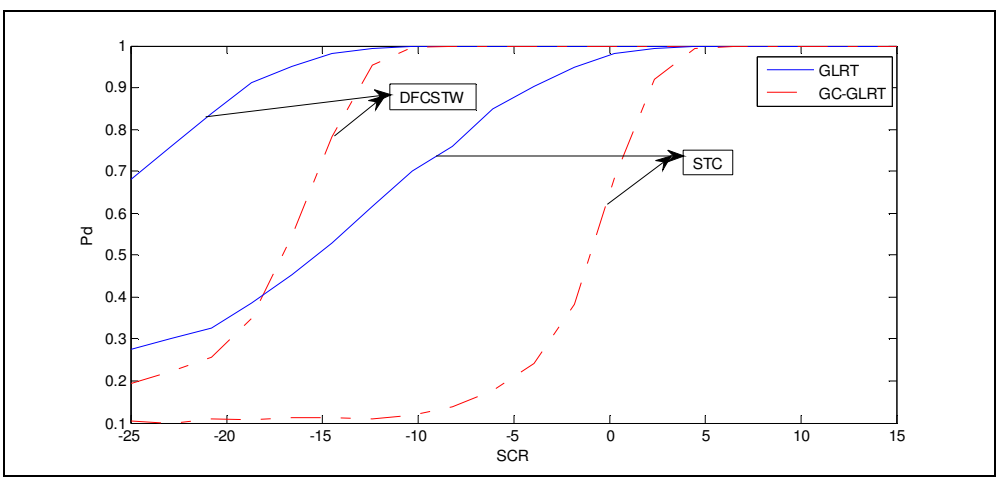

Figure 2. Pd versus SCR plots of GLRT (solid curves) and GCGLRT (dashed curves) receivers with Orthogonal DFCSTW Code set and STC for $\mathrm{Pfa}=10^{-4}, \mathrm{~N}=8, \mathrm{Tx}=4, \mathrm{Rx}=4, \rho=0.9, \mathrm{~K}=64, \mathrm{v}=0.5$ parameter.

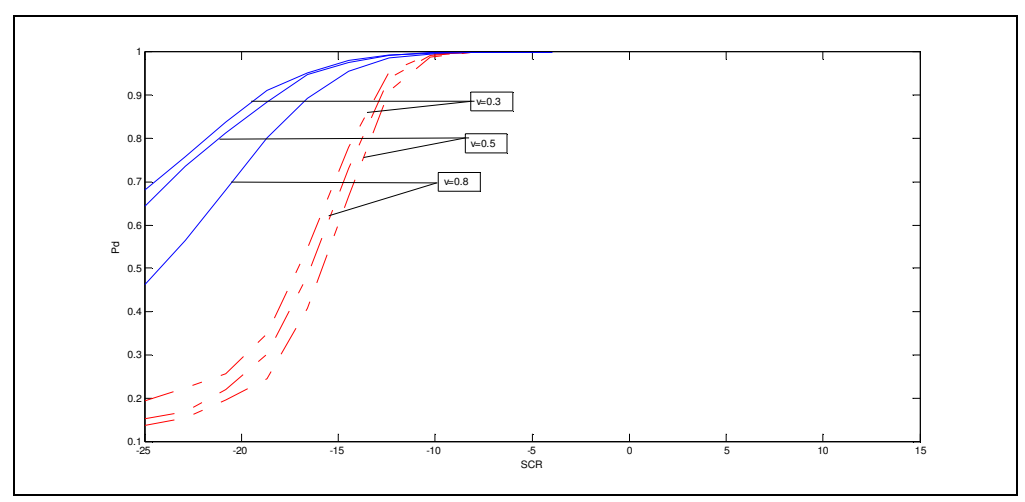

Figure 3. Pd versus SCR plots of GLRT (solid curves) and GCGLRT (dashed curves) receivers with Orthogonal DFCSTW Code set for $\mathrm{Pfa}=10^{-4}, \mathrm{~N}=8, \mathrm{Tx}=4, \mathrm{Rx}=4, \rho=0.9, \mathrm{~K}=64, \mathrm{v}=0.3,0.5$ and 0.8 as variable parameter.

\section{CONCLUSIONS}

In this paper, orthogonal DFCSTW is modelled to transmit in a spikier clutter environment. The results show that the performance of $\mathrm{P}_{\mathrm{d}}$ is better for DFCSTW codes than STC waveform. The results also show that the DFCSTW code has the better performance in spikier clutter. 


\section{REFERENCES}

[1] DJ. Rabideau, P. Parker, (2003) "Ubiquitous MIMO multifunction digital array radar", Proc. Asilomar Conf Signals, Syst., Comput., pp. 1057-64.

[2] D.W. Bliss, K.W. Forsythe, (2003) "Multiple-input multiple-output radar and imaging: degrees of freedom and resolution", Proc. Asilomar Conf. Sig- nals, Syst., Comput., vol. 1, pp. 54-9.

[3] F.C. Robey, S. Coutts, et al, (2004) "MIMO radar theory and experimental re-sults", Proc. Asilomar Conf. Signals, Syst., Comput., vol. 1, pp. 300-304.

[4] Bekkerman, J. Tabrikian, (2006) "Target detection and localization using MIMO radars and sonars", IEEE Trans Sig Proc, vol. 54, pp. 3873-3883.

[5] J. Tabrikian,(2006) "Barankinbounds for target localization by MIMO ra- dars", IEEE Wksh. SensorArray \& Multichannel Sig. Proc., pp. 278-281.

[6] K.W. Forsythe, D.W. Bliss, (2005) "Waveform correlation and optimization issues for MIMO radar", Proc. Asilomar Conf. Signals, Syst., Comput., pp. 1306-1310.

[7] K.W. Forsythe, D.W. Bliss, et al, (2004) "Multiple-input multiple-output radar: performance issues", Proc. Asilomar ConI Signals, Syst., Comput., vol. 1, pp. 310-315.

[8] E.Fishler, A.Haimovich, R. Blum, L.Cimini, D.Chizhik, and R. Valenzuela, (2004) "MIMO radar: An idea whose time has come," in proc. of IEEE International Radar conference, Philadelphia. PA, pp No.71-78.

[9] Hai Deng, (2004) "Discrete frequency-coding waveform design for netted radar systems", in IEEE Signal Proces. letters, vol. 11, Issue 2, pp 179-182.

[10] Bo Liu, Zishu He, Oian He,(2007) "Optimization of orthogonal discrete frequency-coding waveform based on modified genetic algorithm for MIMO radar", in Inter. Conf. on Commun. Circuits and Syst., Kokura, pp no. $966-970$.

[11] Bo Liu, Zishu He, Jun Li, (2008) "Mitigation of autocorrelation sidelobe peaks of orthogonal discrete frequency-coding waveform for MIMO radar", in proc. of IEEE Radar confer., China, Chengdu, pp 16.

[12] Hao He, Petre Stoica, Jain Li, (2009) “ Designing unimodular sequences sets with good correlationIncluding an application to MIMO Radar", in IEEE Trans. on Signal Proces., Vol. 57, No. 11, pp 4391-4405.

[13] Eran Fishler, Alexander Haimovich, Rick S. Blum, Leonard J. Cimini, Dmitry Chizhik, Reinaldo A. Valenzuela., (2006) " Spatial Diversity in Radars-Models and Detection Performance", IEEE Trans. on Sig Proc, Vol. 54, No. 3, pp. 823-837.

[14] Haimovich, A.M., Blum, R.S., Cimini, L.J., (2008) "MIMO Radar with widely separated antennas", in IEEE Sig Proc Magazine, Vol 25, Issue 1, PP No.116-129.

[15] Jian Li, Petre Stoica., (2007) "MIMO Radar with Colocated Antennas" in IEEE Sig Proc Magazine, vol 24, Issue 5, pp No:106-114.

[16] Zengjiankui,Hezishu,Liubo, (2007) “Adaptive Space-time-waveform Processing for MIMO Radar”, in Inter. Conf. on Commun., Circuits and Syst., Kokura, pp no. 641 - 643.

[17] A. Farina, F. Gini, M.V. Greco, L. Verrazzani, (1997) "High resolution sea clutter data: a statistical analysis of recorded live data", IEEE Proc.-Radar, Sonar Navigation 144 (3) page: 121-130.

[18] K.J. Sangston, K.R. Gerlach, (1994) "Coherent detection of radar targets in a non-Gaussian background", IEEE Trans. Aerospace Electron. Systems 30 (2), page: 330-340.

[19] Rui Fa, Rodrigo C. de Lamare , (2010) “Knowledge-aided reduced-rank STAP for MIMO radar based on joint iterative constrained optimization of adaptive filters with multiple constraints", in IEEE Inter. Conf. on Acuost. Speech Sig Proc., Dallas, TX, pp. 2762 - 2765.

[20] Antonio De Maio, Marco Lops, (2007) “Design Principles of MIMO Radar Detectors”, in IEEE Trans. Aero. Elec. Syst., Vol 43, Issue 3, pp. 886-898.

[21] Guolong Cui • Lingjiang Kong • Xiaobo Yang, (2012) "GLRT-based Detection Algorithm for Polarimetric MIMO Radar Against SIRV Clutter”, in Circuits Syst Signal Process, Vol 31, pp.10331048.

[22] Guolong Cui, Lingjiang Kong, Xiaobo Yang,Jianyu Yang, (2010) “Two-step GLRT design of MIMO radar in compound-Gaussian clutter", in IEEE Radar Conf, Washington, DC, pp. 343 - 347.

[23] A. De Maio, C. Hao, D. Orlando, (2014) "An Adaptive Detector with Range Estimation Capabilities for Partially Homogeneous Environment”, in IEEE Sig. Proc. Letters, vol 21, no. 3, pp no- 325-329. 
[24] B. Roja Reddy, M uttarakumari, (2012) "Generation of orthogonal discrete frequency coded waveform using accelerated particle swarm optimization algorithm for MIMO radar", Proceedings of the Second Inter. Conf. on Computer Science, Engg. and App. (ICCSEA 2012), New Delhi, India, Volume 1, pp 13-23.

\section{AUTHORS}

Smt. B. Roja Reddy received the B.E degree in 1998 from Gulbarga University, Karnataka and M. Tech degree in 2004 from VTU, Karnataka.Presently working at R.V. college of Engineering with an experience of 11 years in the teaching field. Her research interest lies in various areas signal Processing. Currently précising her Ph.D in Radar Signal Processing \& MIMO Radar.

Dr. M Uttara Kumari received the B.E degree in 1989 from Nagarujna University, Hyderabad, Andhra Pradesh and M.E degree in 1996from Bangalore University, Karnataka and Ph.D degree in 2007 from Andhra University. Presently working at R.V.College of Engineering with an experience of 19 years in the teaching field. Her research interest lies in various areas of radar systems, Space-time adaptive processing, speech processing and image processing.
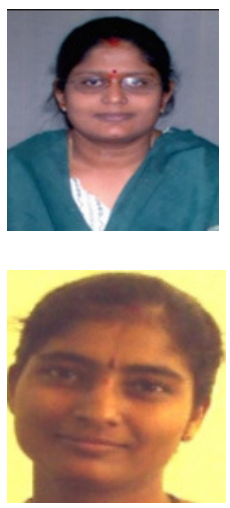